\title{
FDA slow to encourage lupus drugs
}

Systemic lupus erythematosus (SLE) is a complex and unpredictable autoimmune disease for which there have been no fundamental treatment advances since the 1970s. A longstanding lack of consensus on how to demonstrate efficacy prompted a meeting of the US Food and Drug Administration's (FDA; Rockville, MD) Arthritis Advisory Committee on February 23 with academic experts and industry representatives. However, only a partial consensus was reached, leaving companies guessing at endpoints-a risk likely to discourage others from developing drugs for the disease, which affects an estimated 1.4-2.0 million people in the US.

Lupus is caused when autoimmune antibodies bind to a patient's DNA, leading to inflammation, organ damage, and infections. "Clinical benefits in lupus are not well defined at present," says Marc Gurwith, chief medical officer at Genelabs Technologies (Redwood City, CA). Indeed, companies currently have to guess which endpoints will favor the biological mechanisms of their agents and be considered adequately rigorous by FDA.

The obstacles to an easy consensus stem from the unpredictable nature of lupus. Often more than one organ is affected, but in patterns that vary from patient to patient. In addition, the course of "flareups," remission times between flareups, and the overall rate of disease progression are difficult to predict. Consequently, it can be very difficult to enroll a relatively homogeneous patient group or to estimate flareup rates in the control group.

The central debate focuses on whether a drug must improve a patient "globally" or whether efficacy in a specific organ is adequate. Global efficacy is measured by a variety of scoring systems for disease activity, damage, and quality of life, as well as by the ability to reduce doses of steroids, the cornerstone of current therapy. Organ-specific efficacy is measured by the frequency and severity of flareups and by common assays, such as serum creatinine levels, in the case of kidney involvement.

Despite the uncertainty, there are three drugs in clinical trials and two more scheduled to begin efficacy testing this spring. However, most biotechnology companies involved want FDA to draft guidelines for lupus similar to those recently published in February for rheumatoid arthritis (RA), which lay out clear definitions of response to treatment. Reflecting the complexities of RA and the subjectivity of some

David Webber is a freelance writer working in Dobbs Ferry, NY. common measures of benefit, the RA criteria require a combination of physical damage measurements, "global" assessments, and laboratory markers of disease activity. "We should follow the RA model because it would be premature to demand any single endpoint," says Gurwith.

Several speakers at the meeting argued that specific organ indications would be valid because the varying biological mechanisms of novel agents imply specific therapeutic activity and because focusing on patients with involvement of a specific organ allows more patient homogeneity in a clinical trial. But others argued that it would be necessary to track a

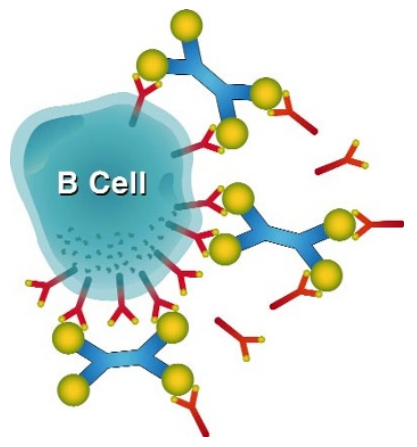

La Jolla's lupus drug (blue) is specific for antiDNA antibodies (red). But the FDA is nonspecific about primary endpoints.

patient "globally" if unanticipated effects were not to be missed. Vibeke Strand of Stanford University remarked that, in the case of lupusassociated nephritis, for example, she would want to track a patient's overall health at the same time as looking at kidney improvements.

Strand reported on the Outcome Measures in Rheumatology Clinical Trials (OMERACT), an academic group that developed a consensus on the most important assessment parameters in lupus trials. The group found disease activity, quality of life, drug toxicity, and organ damage to be the most important.

Although the FDA advisory panel agreed that those parameters should be included in all lupus trials, it did not conclude which alone, if any, would be adequate for drug approval. "We do have agreement on what we need to look at," commented Strand. "We're just not clear yet on how to put it together." This leaves companies having to continue blindly choosing endpoints for clinical trials.

The clinical experience of Genelabs reflects the learning curve down which lupus drug sponsors have been moving in the 1990s. In its initial phase III trial of DHEA (dehydroepiandosterone), the primary endpoint of sustained reductions in steroid use proved problematic because more patients than expected were able to taper steroid use. This probably reflected maintenance doses higher than required to maintain stable disease activity at study entry, making it unclear whether dose reductions reflected changes in disease activity.

In Genelabs' current phase III study, the original primary endpoint was based on reductions in four measures of SLE activity and global response. Concerned that this definition is "first generation," and that these scoring systems could miss some patient benefits, are not routinely used in the clinic, and do not always correlate well with each other, Genelabs has added remission time between (frequency of) flareups of any kind. However, this is complicated by the current lack of a consensus definition of a flareup. Genelabs expects to complete the study this month.

Other companies' strategies appear roughly evenly divided between global and organ-specific endpoints. La Jolla Pharmaceuticals (San Diego, CA) is conducting phase II/III trials of LJP-394 with Abbott Laboratories (Abbott Park, IL). LJP-394, a construct of oligonucleotides linked to a triethylene glycol platform, disrupts autoimmune signaling by binding anti-DNA antibodies (seen in lupus only) on the surface of B cells. The primary endpoint is reduction in the frequency of kidney flares.

IDEC Pharmaceuticals (San Diego, CA) and Biogen (Cambridge, MA) are both developing humanized monoclonal antibodies (Mabs) to CD40 ligand, a protein on activated T cells. By binding CD40 ligand, Mabs block interactions required for the co-stimulation pathway between T cells and B cells, thereby affecting the underlying immune misregulation in lupus.

IDEC is awaiting institutional review board approvals to initiate phase II testing with a primary endpoint of overall disease activity. Biogen, on the other hand, is rumored to have made kidney flareups the primary endpoint in its phase II trial.

In a different approach, Alexion Pharmaceuticals (New Haven, CT) is attempting to reduce tissue-damaging inflammation with its product, 5G1.1, a monoclonal against the C5 complement protein. Alexion plans to begin phase II testing this spring, but has not disclosed its primary endpoint.

Industry representatives remain optimistic that FDA will produce a guidance document eventually_-if not this year, then next. "The lupus field has moved forward," says Steven Engle, La Jolla CEO. "There's more of a coalescing of opinions regarding endpoints now. There was much less agreement a few years ago."

David Webber 